\title{
Regulation of Hematopoietic Stem Cell Self-Renewal and Leukemia Maintenance by the PI3K-mTORC1 Pathway
}

\author{
Joydeep Ghosh ${ }^{1,2} \cdot$ Reuben Kapur ${ }^{1,2}$
}

Published online: 6 October 2016

(C) Springer International Publishing AG 2016

\begin{abstract}
Hematopoietic stem cells (HSC) are the most welldefined stem cell population and have provided invaluable understanding of tissue-specific stem cell development and functions. Aberrant proliferation and differentiation of HSCs cause various hematologic malignancies. Studies have established that within the bulk leukemic population, there are malignant leukemic stem cells (LSCs), which can give rise to leukemia through serial transplantation. LSCs possess similar properties like HSCs, and they can both undergo self-renewal and differentiation. Similarly, HSCs and LSCs both remain in a quiescent state to prevent exhaustion due to proliferation. These processes are tightly regulated by different signaling pathways. Class IA phosphoinositide 3-kinase (PI3K)-mechanistic target of rapamycin complex (mTORC) pathway is a key pathway in regulating HSCs as well as LSCs. In this review, we focus on recent developments regarding the role of different components of the PI3K-mTORC pathway in regulating $\mathrm{HSC}$ and LSC functions.
\end{abstract}

This article is part of the Topical Collection on Role of Classical Signaling Pathways in Stem Cell Maintenance

Reuben Kapur

rkapur@iupui.edu

Joydeep Ghosh

jghosh@iu.edu

1 Department of Microbiology and Immunology, Indiana University School of Medicine, Indianapolis, IN, USA

2 Department of Pediatrics, Herman B Wells Center for Pediatric Research, Indiana University School of Medicine, 1044 W. Walnut Street, R4-168, Indianapolis, IN 46202, USA
Keywords Hematopoietic stem cells $\cdot$ Self-renewal · Leukemia stem cells $\cdot$ mTORC1 $\cdot$ Akt $\cdot$ PTEN

\section{Introduction}

The hematopoietic system has been considered the model paradigm to study the properties and functions of stem cells as well as understanding their contribution to diseases. Hematopoietic cells contribute to different physiological processes as well as protect the body by mounting immune responses against different pathogens. The hematopoietic system is composed of a vast network of cells that are part of a definitive functional hierarchy. The HSCs reside at the apex of this hierarchy and under steady-state conditions; most of the adult HSCs are maintained in the quiescent state $[1,2]$. In response to various signals, HSCs differentiate to multipotent progenitors, which in turn, progressively differentiate into more lineage-committed effector cells. In order to maintain hematopoiesis throughout ones' lifetime, the HSCs have to undergo self-renewal, differentiation, and proliferation and maintain a distinct metabolic profile. These processes are regulated by a host of signaling networks, and deregulation of these networks results in various hematologic malignancies. Hematologic malignancies arise from aberrant proliferation and differentiation of hematopoietic cells and can be initiated by LSCs. LSCs are functionally defined as the leukemic cells, which can propagate disease through serial transplantation. LSCs have been implicated in the persistence of acute myeloid leukemia (AML) and relapse in patients [3]. Like HSCs, LSCs have the ability to self-renew and differentiate, and maintenance of LSCs is dependent on their metabolic profile $[4,5]$. One of the pathway that has been identified as a key player in the regulation of HSCs and LSCs is the PI3K-Akt-mTORC pathway. 


\section{Components of the PI3K-Akt-mTORC1 Pathway}

Mechanistic target of rapamycin (mTOR) is a serine/threonine complex that can be divided into two distinct complexes, mTOR complex 1 (mTORC1) and mTOR complex 2 (mTORC2) [6]. mTORC1 activity is primarily regulated by class IA phosphoinositide 3-kinases (PI3Ks) [7]. PI3Ks are dimers formed by regulatory (p85 $\alpha, \mathrm{p} 85 \beta, \mathrm{p} 55 \alpha, \mathrm{p} 55 \gamma$, and $\mathrm{p} 50 \alpha)$ and catalytic (p110 $\alpha, \mathrm{p} 110 \beta$, and p1108) subunits [8]. Following activation by upstream signals, which include growth factors, nutrient status, and oxygen level, PI3K is recruited to the cell membrane where it converts phosphatidylinositol $(4,5)$-bisphosphate (PIP2) to phosphatidylinositol (3,4,5)-triphosphate (PIP3) [7] (Fig. 1). PIP3 recruits 3-phosphoinositide-dependent protein kinase-1 (PDK1) and Akt to the plasma membrane where PDK1 phosphorylates T308 and activates Akt [9] (Fig. 1). The primary negative regulators of PI3K-mTORC1 pathway are phosphatase and tensin homolog (PTEN), tuberous sclerosis 1 (TSC1 or tuberin), and tuberous sclerosis 2 (TSC2 or harmatin). PTEN converts PIP3 to PIP2 and subsequently inhibits the recruitment of PDK1 to the plasma membrane [10-12]. TSC2 is a GTPase-activating protein [13], and it is associated with TSC1 for inactivating the small $\mathrm{G}$ protein Ras homolog enriched in brain (Rheb) [14]. Akt phosphorylates TSC2, which results in binding of TSC2 to 14-3-3 and subsequent reduction in GAP activity of the TSC1/TSC2 complex [15-17]. Withdrawal of TSC-mediated inhibition results in increased GTP-bound Rheb levels [14, 15] (Fig. 1). GTPbound Rheb phosphorylates and subsequently activates mTORC1. Activated mTORC1 phosphorylates the translational regulators eukaryotic translation initiation factor $4 \mathrm{E}$ (eIF4E)-binding protein 1 (4E-BP1) and ribosomal protein S6 kinase 1 (S6K1). Hypophosphorylated 4E-BP1 binds to eIF4E and prevents its interaction with eIF4G. Phosphorylation of 4E-BP1 results in dissociation from eIF$4 \mathrm{G}$ and subsequent initiation of translation by recruitment of eIF-4G to the $5^{\prime}$ cap structure of mRNA. S6K1 is phosphorylated on Thr-389 residue by mTORC1 followed by phosphorylation on Thr-229 by PDK-1 [18-20]. mTORC1 also regulates mTORC2 activation through activating S6K1. Following activation by mTORC1, S6K1 inhibits mTORC2-dependent Akt activation on Ser-473 (Fig. 1). S6K1 phosphorylates rictor on Thr-1135 and mSIN1 on Thr-86 and Thr-398, which results in reduced phosphorylation of Akt on Ser-473 [21, 22]. Apart from mTORC1, activation also regulates the activity of Foxo group of proteins. The Foxo group of transcription factors regulate transcription of genes involved in cell-cycle

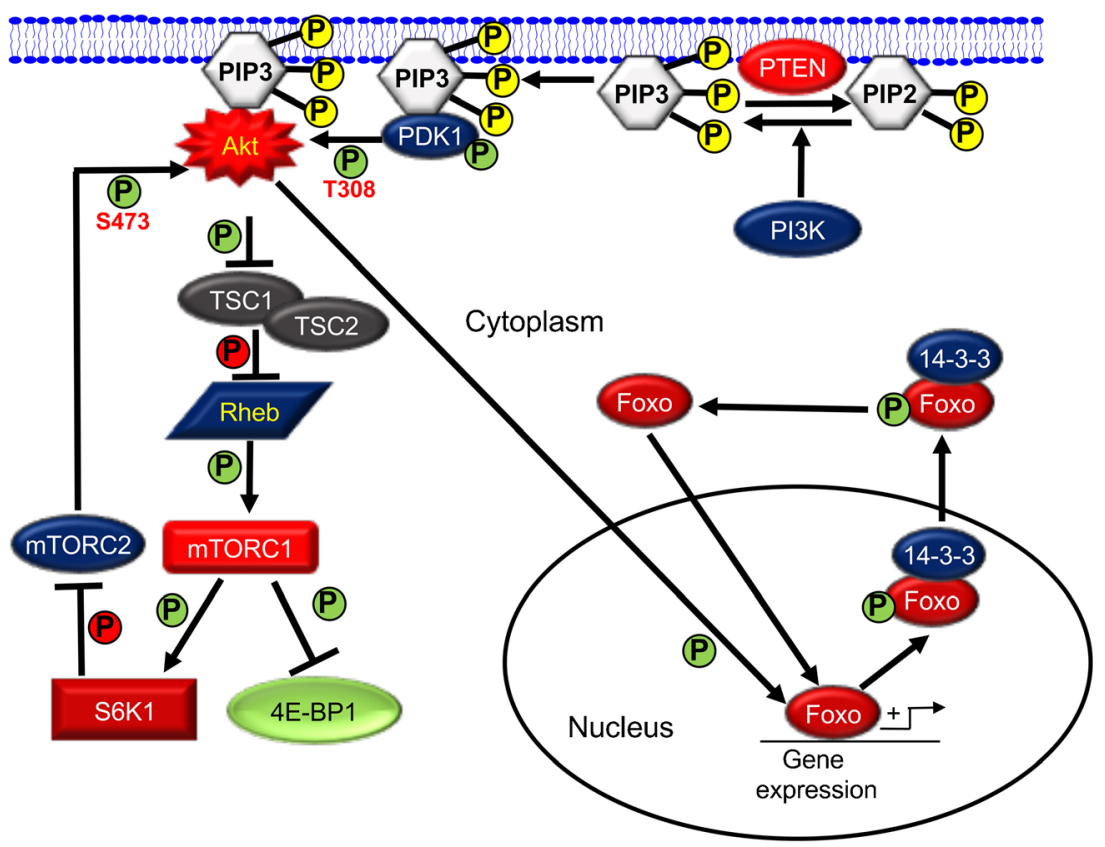

Fig. 1 The class IA phosphoinositide 3-kinase (PI3K)- mechanistic target of rapamycin complex (mTORC) signaling pathway. In response to growth factors and mitogenic stimuli, phosphatidylinositol $(4,5)$ bisphosphate (PIP2) is converted into phosphatidylinositol $(3,4,5)$ trisphosphate (PIP3) by phosphoinositide 3-kinases (PI3K) activity. PTEN acts as a negative regulator of mTORC activity by converting PIP3 to PIP2. Akt binds to PIP3 allowing 3-phosphoinositide dependent protein kinase-1 (PDK1) to access the activation sites of Akt. Following phosphorylation by PDK1, activated Akt phosphorylates and inhibits tuberous sclerosis 2 (TSC2)-mediated inhibition of Rheb.
Activated Rheb subsequently phosphorylates and activates mTORC1. Activated mTORC1 phosphorylates the translational regulators eukaryotic translation initiation factor 4E (eIF4E)-binding protein 1 (4E-BP1) and ribosomal protein S6 kinase 1 (S6K1). On the other hand, mTORC2 acts an activator of Akt by phosphorylating it on Ser473. S6K1 exerts an inhibitory effect on mTORC2 thus negatively regulating Akt phosphorylation. Foxo family of transcription factors translocate to nucleus and regulate gene expression. Akt phosphorylates Foxo group of transcription factors and facilitates their nuclear export by sequestering them within the cytoplasm 
arrest, apoptosis, and reactive oxygen species (ROS) detoxification. Activated Akt phosphorylates Foxo group of proteins on highly conserved sites. In the nucleus, phosphorylation of Foxos creates a binding site for the chaperon protein 14-3-3. Binding of 14-3-3 to Foxos masks their nuclear localization signal (NLS), thus facilitating their removal from the nucleus and suppressing Foxo dependent gene transcription [23] (Fig. 1). Binding of 14-3-3 to Foxos also exposes their nuclear export signals and promotes export of Foxos from the nucleus to the cytoplasm [24].

\section{Effect of Inhibition of mTORC1 Activity on HSCs}

Inhibition of PI3K pathway has been shown to cause substantial functional defects in HSCs. Deficiency of p $85 \alpha$ subunit of PI3K complex in fetal liver HSCs results in reduced long-term engraftment and differentiation of HSCs (Table 1) [25]. Deficiency of either Akt or Akt2 does not impair HSC development and functions suggesting a redundancy of these isoforms of Akt in HSCs. However, deficiency of both Akt1 and Akt2 in fetal liver HSCs as well as in adult young HSCs also results in reduced engraftment in peripheral blood of recipients following serial transplantation (Table 1) [26]. Myeloid and lymphoid lineages are also reduced in Akt1- and Akt2deficient HSC recipients compared to control [26]. Akt1- and Akt2-deficient HSCs have increased quiescence level, and transition of HSCs from the G0 to G1 phase of cell cycle is impaired (Table 1) [26]. Downstream of Akt, deficiency of mTOR, a common component of mTORC1 and mTORC2, causes significant reduction in engraftment potential of HSCs [27]. Raptor, a specific component of mTORC1, is expressed in HSCs. Raptor deficiency increases the frequency of shortterm HSCs and progenitors in bone marrow, while the frequency of long-term HSCs (LT HSCs) remains unaffected.
However, raptor-deficient HSCs have reduced ability to reconstitute lethally irradiated recipients following transplantation and they localize further from osteoblast cells inside the bone marrow [28]. In a competitive assay, raptor-deficient HSCs show reduced engraftment potential compared to controls (Table 1). The role of rictor, a specific component of mTORC2, on HSC function has also been described in multiple studies. Two studies have demonstrated that conditional deletion of rictor causes a decrease in engraftment of HSCs following transplantation [28, 29], which suggests that mTORC2 is required for engraftment and self-renewal of HSC. Downstream of mTORC1, S6K1 has also been identified as a key regulator of HSC maintenance and function. S6K1 deficiency results in reduced number of HSCs. S6K1deficient HSCs also have reduced quiescence and p21 expression level. Furthermore, S6K1 deficiency also impairs the self-renewal ability of HSCs (Table 1) [30••].

In contrast to genetic approaches, pharmacological inhibition of mTORC1 by rapamycin has yielded opposite results. Inhibition of mTORC1 activity in old HSCs by administering rapamycin results in increased long-term engraftment of HSCs [31]. Treatment of ex vivo expanded murine or human HSCs by rapamycin also results in increase in long-term engraftment in transplant recipients [32, 33]. However, another study showed murine c-Kit+ cells cultured in presence of rapamycin fail to engraft in lethally irradiated recipients [34]. The difference between pharmacological and genetic approaches to inhibit PI3K-mTORC1 activity might be due to the duration of inhibition. In genetic approaches, the prolonged inhibition causes HSC to behave differently than in studies using pharmacological inhibitors. Short-term inhibition of PI3K-mTORC1 pathway by pharmacological inhibitors might cause expansion of functional LT-HSCs in culture. In mice, HSCs undergo proliferation until 3 weeks after birth

Table 1 Role of effectors of PI3K-mTORC1 pathway in HSC function

\begin{tabular}{|c|c|c|c|c|c|c|}
\hline Protein & Model & $\begin{array}{l}\text { Activator/inhibitor } \\
\text { of mTORC pathway }\end{array}$ & Cell cycle & $\begin{array}{l}\text { Long-term engraftment } \\
\text { in primary recipients }\end{array}$ & $\begin{array}{l}\text { Serial } \\
\text { transplant }\end{array}$ & Self-renewal \\
\hline PTEN (35) & Mx1-Cre & Inhibitor & Increased & Decreased & - & - \\
\hline PTEN (37) & Scl-Cre, Mx-Cre & Inhibitor & Unchanged & - & - & - \\
\hline $\mathrm{p} 85 \alpha(25)$ & $\mathrm{KO}$ & Activator & Decreased & Decreased & - & - \\
\hline Akt (26) & $\mathrm{KO}$ & Activator & Decreased & Unchanged & Tertiary & Decreased \\
\hline TSC1 (39) & Mx1-Cre & Inhibitor & Increased & Decreased & - & - \\
\hline Rheb2 (40) & Overexpression & Activator & Increased & Decreased & Secondary & Decreased \\
\hline mTOR (27) & Mx1-Cre & Activator & Increased & Decreased & - & - \\
\hline Raptor (28) & Mx1-Cre & Activator & Unchanged & Decreased & - & - \\
\hline Rictor (28) & Mx1-Cre & Increased & - & Decreased & - & - \\
\hline Rictor (29) & Mx1-Cre & Activator & Unchanged & Unchanged & Secondary & Decreased \\
\hline S6K1 (30) & $\mathrm{KO}$ & $\begin{array}{l}\text { Downstream substrate } \\
\text { of mTORC1 }\end{array}$ & Increased & Unchanged & Tertiary & Decreased \\
\hline PML (41) & $\mathrm{KO}$ & Inhibitor & Increased & Decreased & Secondary & Decreased \\
\hline
\end{tabular}


and return to a more quiescent state by week 4 suggesting agespecific differential requirements of factors regulating quiescence of HSCs [1]. HSCs might require differential mTORC1 activity at different stages of development. Moderate level of mTORC1 activation might be required at early stages to sustain self-renewal and expansion of LT-HSCs. However, mTORC1 activation at later stages could impair the selfrenewal potential of HSCs.

\section{Effect of Hyperactivation of PI3K Pathway on HSCs}

In addition to the inhibition of the PI3K pathway, hyperactivation of components of this pathway also results in severe functional defects in HSCs. Conditional deletion of PTEN increases the proliferation and mobilization of adult HSCs [35]. Furthermore, PTEN-deficient adult HSCs display a reduction in engraftment following 6 weeks after transplant. PTEN deletion causes increase in hyperactivation of PI3K pathway in adult HSCs and MPPs. Phosphorylation of Akt at Ser-473 is upregulated in PTEN-deficient adult HSCs, which suggests that PTEN regulates mTORC2 signaling in HSCs. Deletion of rictor causes attenuation of Akt phosphorylation in PTEN-deficient HSCs (Fig. 2). Moreover, rictor deficiency also leads to reduced proliferation and increased engraftment of PTEN-deficient HSCs [29]. Treatment with rapamycin, but not rictor deletion, attenuates the mobilization of WT HSCs [29]. However, deletion of rictor, but not inhibition of mTORC1, reduces mobilization of HSCs from the bone marrow to the spleen after treatment with G-CSF/cyclophosphamide [29]. These results suggest that mTORC1, but not mTORC2, is the major mediator of HSC mobilization under physiological conditions. However, following loss of PTEN, mTORC2 acts as the regulator of HSC proliferation and mobilization from the bone marrow [29].

Loss of PTEN also results in upregulation of tumor suppressor signaling in HSCs to deplete HSCs in order to prevent leukemogenesis. Expression levels of p16 and p53, both tumor suppressors, are significantly increased in PTENdeficient HSCs. Deficiency of either p16 or p53 increases the engraftment of PTEN-deficient HSCs [36]. Recent evidences suggest that PTEN differentially regulates neonatal and adult HSCs. Conditional deletion of PTEN does not affect the cycling and mobilization of neonatal HSCs. Furthermore, following transplantation, PTEN-deficient HSCs derived from neonates can engraft in recipient up to 16 weeks. The difference in functional properties of PTEN-deficient neonatal and adult HSCs could be attributed to the lack of PI3K-AktmTORC1 hyperactivation in neonatal HSCs following

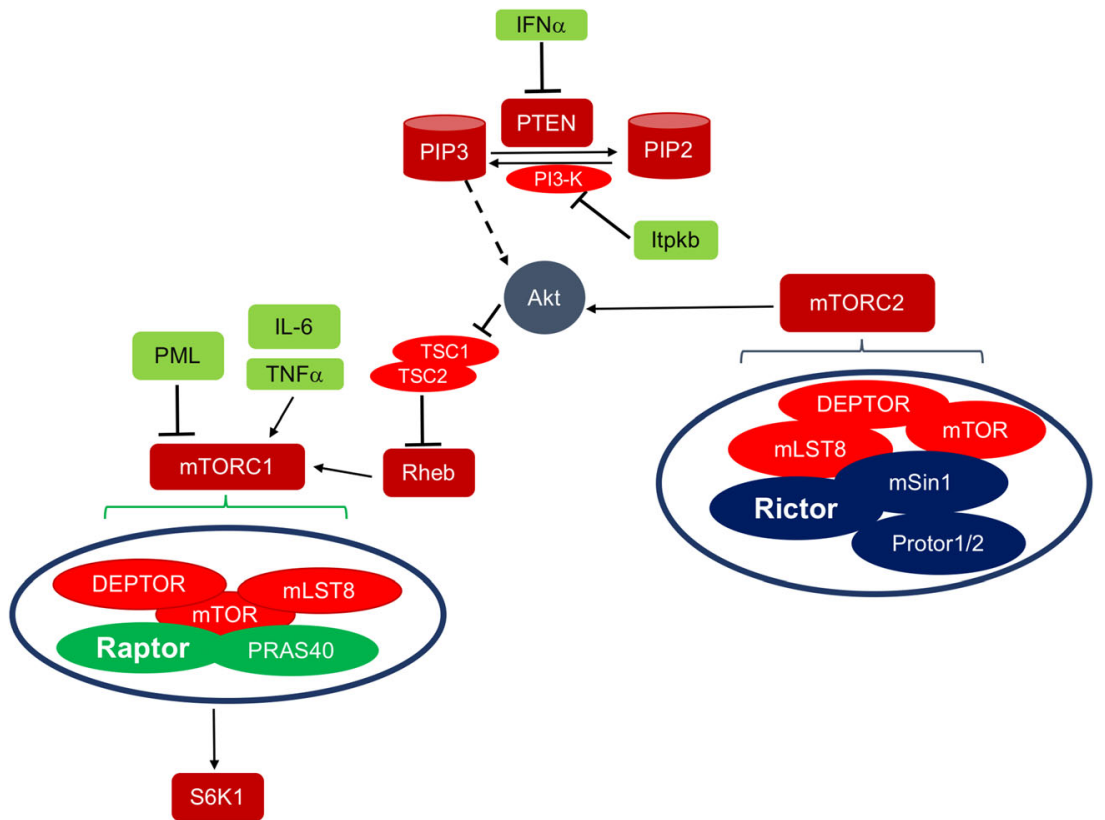

Fig. 2 PI3-K-mTORC1 signaling in hematopoietic stem cells. mTORC1 has five and $\mathrm{mTORC} 2$ has six protein components. Both complexes share the catalytic mTOR subunit, mammalian lethal with sec-13 protein 8 (mLST8), DEP domain containing mTOR-interacting protein (DEPTOR). Regulatory-associated protein of mammalian target of rapamycin (raptor) and proline-rich Akt substrate $40 \mathrm{kDa}$ (PRAS40) are specific to mTORC1, whereas rapamycin-insensitive companion of mTOR (rictor), mammalian stress-activated map kinase-interacting protein $1(\mathrm{mSin} 1)$, and protein observed with rictor 1 and 2 (protor $1 / 2$ ) is part of mTORC2 complex. In HSCs, rictor phosphorylates and activates Akt on Thr-308 residue. Itpkb exerts its inhibitory effect on
PI3K-mTORC1 pathway by inhibiting PI3K activity. In absence of Itpkb in HSCs, Akt-mTORC1 pathway becomes hyperactivated. Inflammatory mediators like IFN- $\alpha$, TNF- $\alpha$, and IL- 6 act as activators of mTORC1. IFN- $\alpha$ inhibits PTEN activity thus increasing Akt-mediated mTORC1 activation in HSCs. In response to inflammatory stress, TNF- $\alpha$ and IL- 6 activates mTORC1, which results in increased cycling of HSCs. PML sequesters mTORC1 to nuclear bodies thus acting as an inhibitor of mTORC1 activity. In absence of PML, mTORC1 activity is increased in HSCs, which corresponds with increased cycling and diminished repopulating ability 
PTEN deletion. PTEN-deficient neonatal HSCs and MPPs do not show any changes in the activation of PI3K pathway, which suggests that PTEN does not regulate this pathway in neonate HSCs [29].

In these studies, the mice were in C57BL/6 background and had interferon- $\alpha$ (IFN- $\alpha$ ) inducible $\mathrm{Mx} 1$ promoter to delete PTEN in HSCs [29, 35, 36]. In a tamoxifen (Tx)inducible model of PTEN deletion in mice with mixed genetic background, contrasting results were observed. Conditional deletion of PTEN did not cause any increase in proliferation of HSCs in the Tx-inducible model of PTEN deletion [37]. Moreover, the study also could not detect any changes in Akt phosphorylation in HSCs following PTEN deletion. However, in the same study, mice with mixed genetic background and expressing Mx1 promoter also did not show any change in HSC quiescence following PTEN deletion. Although PTEN-deficient HSCs have reduced frequency of functional HSCs, they showed similar engraftment potential compared to controls [37]. The fact that deletion of PTEN by inducing Mx1 promoter yields different functional outcome in different genetic backgrounds underscores the impact of genetic backgrounds on regulating $\mathrm{HSC}$ development and function.

Downstream of PTEN increase in Akt activity causes dysfunction in HSCs [38], and HSCs bearing constitutively active Akt display reduced engraftment following transplantation. Murine HSC-enriched population (Lin- Sca1+ c-Kit+) undergoes increased proliferation along with increased apoptosis following expression of constitutively activated Akt [38]. However, Akt phosphorylation level is not significantly upregulated when WT HSC undergo rapid proliferation, which suggests that Akt activation might be dispensable in cycling HSC [29]. It could be possible that retroviral expression resulted in significantly increased level of activated Akt compared to physiological level of activated Akt present in WT HSC, which had driven the HSC into increased cycling [38]. This suggests that Akt activation might not be required for proliferation of WT HSCs. Conditional deletion of TSC1, a negative regulator of $\mathrm{mTORC} 1$, leads to hyperactivation of mTORC1 activity in HSCs. TSC1-deficient HSCs undergo increased cycling, along with increased ROS levels, increased mitochondrial biogenesis and have decreased engraftment potential (Table 1) [39]. In HSCs, overexpression of Rheb2, an activator of mTORC1, results in increased cycling and reduced self-renewal (Table 1) [40]. Promyelocytic leukemia protein (PML), a tumor suppressor, has also been identified as a negative regulator of PI3K pathway, and it regulates the activation of Akt and mTORC1 (Fig. 2). Deletion of PML in HSCs results in increased cycling along with a decrease in self-renewal [41]. The quiescent level in Pml-/- HSCs can be restored by inhibiting mTORC1 activity [41]. Overall, hyperactivation of $\mathrm{PI} 3 \mathrm{~K}-\mathrm{mTORC} 1$ results in impaired quiescence and self-renewal of HSCs.

\section{Role of PI3K-mTORC1 Pathway in HSCs Following Inflammatory Responses}

The PI3K-Akt-mTORC1 axis regulates signaling of HSCs in response to inflammatory mediators. Tissue inhibitor of metalloproteinase 1 (TIMP-1) acts as a regulator for expansion of human hematopoietic stem and progenitor cell (HSPC). Stimulation of CD34+ HSPCs by TIMP-1 increases the phosphorylation of Akt at the Thr-308 residue, the target of PI3Kmediated Akt activation. Furthermore, pharmacological inhibition of PI3K activity blocks TIMP-1-mediated expansion of human HSPCs [42]. Inositol-trisphosphate 3-kinases (Itpk) phosphorylate inositol (1,3,4,5)-tetrakisphosphate (IP4), which causes subsequent inhibition of Akt activity (Fig. 2) [43]. Among three isoforms of Itpk, HSCs express only the Itpkb isoform. Itpkb-deficient LT-HSCs display increased cytokine induced Akt activation. Rapamycin treatment reduces hyperproliferation of Itpkb-/- LT-HSCs as well as decreases mTORC1 activity [44]. Deficiency of Itpkb results in increased absolute number of LT-HSCs and MPPs within the bone marrow [44]. The increase in LT-HSC number is associated with a subsequent decrease in quiescent HSCs in Itpkb $-/-$ mice [44]. Itpkb deficiency also results in deficiency of long-term self-renewal ability of HSCs [44]. Itpkb-/- mice develop hematopoietic disorders with progressive aging, which includes reduced LT-HSCs, MPPS, and HPCs in bone marrow and succumb to anemia [44]. In vivo inflammation also results in increased mTORC1-S6K1 activity in HSCs, which causes reduced bone marrow cellularity and reduction in the functional potential of HSCs [45]. Treatment with rapamycin reverts the defects in HSCs caused by inflammation, which suggests that hyperactivation of mTORC1 negatively regulates HSC function following inflammatory stress [45]. IFN $\alpha$, a mediator of inflammation, causes loss of quiescence followed by increased cycling of HSCs [46], which corresponds to a reduction in PTEN expression along with an increase in Akt phosphorylation (Fig. 2). The inflammatory mediators, while mounting an inflammation response, could force the HSCs to proliferate in order to generate more myeloid cells to increase cells regulating short-term innate immunity. As PI3K-mTORC1 pathway is a critical regulator of HSC proliferation under stead-state, inflammatory mediators cause hyperactivation of this pathway to increase HSC proliferation. However, this also compromises the engraftment and self-renewal properties of HSCs.

\section{Role of PI3K-mTORC1 Pathway in Hematologic Malignancies and in Maintenance of LSCs}

The PI3K-mTORC1 pathway is hyperactivated in a large group of AML patients [47-51]. AML patients with low level of phosphorylated Akt in peripheral blood and bone marrow cells have significantly increased median survival time 
compared to patients with increased phosphorylated Akt level (59 weeks vs. 30 weeks) [52]. In patients who are $\geq 60$ years old or diagnosed with secondary AML, phosphorylation of Akt has been associated with lack of response to induction chemotherapy [53]. The PI3-K pathway in AML cells can also be hyperactivated because of activation of the IGF-1/IGF-1R autocrine loop [51].

The $\mathrm{p} 85$ regulatory subunit of PI3K regulates c-Kit D814V mutation induced transformation in murine cells and inhibition of p85 $\alpha$ activity in oncogenic c-Kit-expressing cells is sufficient to suppress growth and leukemogenesis [54, 55]. Consistently, pharmacologic inhibition of mTORC1, a downstream effector of PI3K, activity is sufficient to inhibit the growth of oncogenic c-Kit-expressing cells [54]. The p110 catalytic subunit of PI3K is the primarily upregulated PI3-K isoform in AML cells [56]. Treatment of AML cells with a combination of BYL-719 (a p110 $\alpha$ subunit inhibitor) and OSI-027 (a dual mTOR inhibitor) results in increased cell death compared to treatment with any single of these two drugs [57].

Dysregulated PTEN transcript level in AML patients has been associated with increased activation of Akt and poor prognosis in AML patients [58]. Older AML patients, with cells deficient in PTEN expression but positive for the expression of CD44, demonstrate poor overall survival [59]. In adult mice, deletion of PTEN, a negative regulator of PI3K-pathway, results in AML and T-ALL [35]. Pharmacological inactivation of mTORC1 activity in PTEN-deficient mice prolongs their survival, which suggests that PTEN-deficient AML cells require mTORC1 for leukemogenesis [35]. Studies involving genetic approaches have further elucidated the roles of mTORC1 and mTORC2 in PTEN-loss-evoked leukemogenesis. In PTEN-deficient HSCs, deletion of either raptor or rictor increases the median survival time of mice. However, these mice still display signs of myeloproliferative disorder [28, 29]. Interestingly, neonatal mice do not develop T-ALL when PTEN is deleted in HSCs [29].

Akt is phosphorylated at Thr-308 and Ser-473 in 50-72\% of AML patients $[58,60]$. Phosphorylation on either of the residues of Akt is associated with poor overall survival in AML patients. Downstream of Akt, Rheb1 has been found to be hyperactivated in AML patients [61]. In a murine model, deletion of Rheb1 in AML cells impairs the functional ability of LSC and increases mean survival time [61]. In absence of Rheb1, AML cells undergo increased cell cycle arrest and apoptosis. Negative cell cycle regulatory genes are also upregulated in Rheb1-deficient AML cells. Following deletion of raptor, a component of mTORC1, in vivo progression of AML was delayed. [62]. Deficiency of raptor induces apoptosis selectively in differentiated AML cells but not in undifferentiated AML cells, which might have caused the delay in progression of AML in mice [62]. Moreover, activating phosphorylation level of S6K1 in raptor-deficient AML cells was significantly decreased compared to WT AML cells [62].
Inhibition of S6K1 activity does not affect the initiation of AML. However, S6K1 is required for maintenance of selfrenewal potential of leukemia-initiating cells. In contrast to HSCs, LSC-enriched population have increased quiescence in absence of S6K1 [30••].

There is also evidence suggesting that oncogenes could regulate mTORC1 activation by bypassing Akt activation. Cells bearing FMS-like tyrosine kinase-3 internal tandem duplication (FLT3-ITD) have increased level of activated Stat5, which results in $\mathrm{mTORC} 1$ activation, rendering the cells resistant to PI3K-Akt inhibitor [63]. This study suggests that in AML cells, oncogenic mutations could activate mTORC1 through a mechanism which is independent of Akt activation.

Although there are evidences of hyperactivation of mTORC1 activity in AML, clinical trials with pharmacological inhibitors of mTORC1 have failed to demonstrate broad activity in AML patients. Additionally, the prognostic value of hyperactivated PI3K-mTORC1 pathway in hematologic malignancies depends on the nature and stage of the diseases. AML patients with hyperactive Akt had an increased overall survival and increased relapse free survival compared to patients with reduced Akt phosphorylation at Ser-473 [60]. Increased PI3K activity in immature leukemic cells results in increased number of cells in $\mathrm{S}$ phase of cell cycle, which makes the cells more susceptible to chemotherapeutic agents [60]. In a chronic myeloid leukemia (CML) model of Bcr-Abl driven leukemia, Akt activity was shown to be suppressed in LSCs, which resulted in nuclear localization of Foxo3a [64]. LSCs are able to evade stress-induced depletion in a Foxo3adependent manner [64]. In contrast, in non-LSC population of CML, inactivation of Akt results in increased Foxo3a activity, which causes cell cycle arrest and apoptosis [64]. In MLLAF9-expressing AML cells, constitutive activation of Akt results in growth arrest and also causes increased maturation of cells toward myeloid lineage [65].

The PI3K-mTORC1 pathway might regulate the LSC population by regulating the functions of the Foxo group of proteins. Multiple studies have shown that Foxo group of proteins are a key regulator of propagation of CML and AML [64, 65]. mTORC1 is a critical regulator of Foxo proteins. Foxo3deficient mice display increased ROS production in hematopoietic progenitor cells, which was associated with increased Akt-mTORC1 activation. Depletion of Foxo1, Foxo3, and Foxo4, which act downstream of Akt, results in diminished LSC activity. Following serial transplantation of AML cells, deficiency of Foxos results in increased median survival compared to control groups [65]. Increased proliferation of myeloid cells has been associated with increased intracellular ROS level [5]. Moreover, increased ROS level have been associated with acquisition of DNA damage and cellular apoptosis [66]. AML cells expressing MLL-AF9 maintain a reduced ROS level [5, 64]. Increased ROS level in MLL-AF9-expressing cells results in DNA damage, which was associated with 
downregulation of antioxidant genes regulated by Foxo group of proteins $[64,67]$. It is probable that similar to hematopoietic cells, in Foxo3a-deficient AML cells, ROS level was increased due to upregulation of Akt-mTORC1 activity. However, Akt-driven Foxo3a activity could also regulate self-renewal potential of LSCs differentially depending on the type of leukemia. For example, Foxo3a regulates selfrenewal potential of LSCs in chronic phase CML but not in blast crisis CML. Deletion of Foxo3a impairs the self-renewal ability of Bcr-Abl-expressing cells. However, in cells expressing both Bcr-Abl and NUP98-Hoxa9, deficiency of Foxo3a does not affect the self-renewal potential of LSC [64]. Overall, these studies suggest that the role of PI3K-mTORC1 pathway in hematologic malignancies should be considered differentially based on the type of and stage of the leukemia.

PI3K-mTORC1 pathway has also been identified as a key pathway that synergizes along with other pathways to maintain LSCs. Treatment of Bcr-ABL-expressing cells with arsenic trioxide results in degradation of PML, a negative regulator of PI3K-mTORC1 pathway and increased cellular proliferation of LSCs. This increase in cellular proliferation is more in LSCs compared to HSCs, which increases their susceptibility to AraC and eradicates LSCs [41]. mTORC1 activity is required to induce AMP-activated protein kinase (AMPK) activation mediated cytotoxicity in AML cells. Hyperactivation of mTORC1 by disrupting TSC initiates the unfold protein response in AML cells, which causes increased cytotoxicity following activation of AMPK. However, activation of AMPK does not induce cytotoxicity in normal hematopoietic cells. These data led to a proposed model in which AMPK and mTORC1 are involved in a synthetic lethal interaction in AML and downregulation of mTORC1 activity might be critical for the survival of AML cells [68]. Circadian rhythm is another key physiologic process that is required to maintain LSCs. Bmal1 is a transcription factor and a key player in regulating molecular clock in mammals [69, 70]. Bmall is required for maintenance of LSCs as deficiency of Bmal1 prolongs the survival of mice following secondary transplantation [71]. Circadian oscillation of cellular protein translation is dependent on Bmall activity and regulated by S6K1, the downstream substrate of mTORC1. S6K1 rhythmically phosphorylates Bmall to regulate circadian protein translation. S6K1-mediated phosphorylation of Bmal1 is necessary to associate with the translation machinery and promote translation [72]. Thus, mTORC1 activity might be critical to sustain protein translation in LSCs and their maintenance. Together, these studies indicate that mTORC1 activation as a therapeutic target could be exploited in LSCs without affecting the function of normal HSCs. However, detailed studies are required to determine the physiologic processes that are regulated by the PI3K-mTORC1 pathway in LSCs and the potential effect of targeting specific molecules downstream of mTORC1 in LSCs.

\section{Role of PI3K-mTORC1 Pathway in Regulating Metabolism in HSCs and Leukemic Cells}

Multiple studies have established the importance of metabolic processes in regulation of hematopoiesis and leukemia. Pyruvate, the glycolytic end product, could undergo two potential conversions. Through anaerobic glycolysis, it can be converted to lactate and this step generates two ATPs per molecule of glucose. Pyruvate can be converted aerobically to acetyl-CoA for mitochondrial metabolism. This step generates 36 ATPs per molecule of glucose. Under steady-state, HSCs as well as other hematopoietic subsets have very low level of tricarboxylic acid cycle (TCA cycle), which indicates their dependence on glycolysis to sustain cellular metabolism [73.0. Compared to the hematopoietic progenitors or terminally differentiated cells, HSCs display a significant increase in pyruvate level which corresponds with low ATP level it gradually increases as HSCs differentiate [73••]. The conversation of pyruvate to acetyl-CoA is catalyzed by pyruvate dehydrogenase (PDH). The PDH complex have three catalytic subunits, pyruvate dehydrogenase (E1), dihydrolipoamide transacetylase (E2), and dihydrolipoamide dehydrogenase (E3) [74]. PDH kinases (Pdks) phosphorylate PDHs and inhibit their activity. Pdks are highly expressed in murine LTHSCs and PDH-E1 is highly phosphorylated in LT-HSCs compared to hematopoietic progenitors or mature cells indicating suppressed PDH activity in HSCs [73••]. Deficiency of Pdk2 and Pdk4 leads to decreased quiescence, engraftment and self-renewal of HSCs along with a decrease in cellular pyruvate level [73••]. In HSCs, Pdks suppress the influx of glycolytic metabolites into mitochondria thereby restricting generation of ATPs and maintaining metabolic quiescence. In HSCs, increased Akt and mTORC1 activities have been associated with decreased glycolysis and reduced expression of Pdk2 and Pdk4 [75]. However, the exact role of PI3KmTOTRC1 pathway in regulating the metabolic profile of HSCs has largely been unknown so far.

AML cells are also highly dependent on glycolysis for cellular metabolism [76]. AML blast cells with high glycolysis rate are resistant to chemotherapeutic agent induced apoptosis [76]. MLL-AF9-expressing AML cells are dependent on glucose metabolism and disruption of glucose metabolism by metabolic stress diminishes the activity of LSCs [5]. In PTEN-deficient hematopoietic cells, inhibition of S6K1 activity results in decreased glycolysis and induces apoptosis [77]. S6K1 is also a positive regulator of glycolysis in Bcr-Ablexpressing cells [78]. However, inhibition of S6K1 activity in Bcr-Abl-expressing cells did not result in induction of apoptosis in the cells [78]. In primary AML cells, stromaderived CXCL12 promotes mTORC1 signaling, which results in increased glycolysis in AML cells [79]. However, clinical trials with 2-deoxy-D-glucose (2DG), a pharmacological inhibitor of glycolysis, have been largely ineffective [80, 81]. 
Treatment of cancer cells with 2DG reprograms their metabolic profiles via mTORC1-dependent upregulation of multiple metabolic pathways including oxidative phosphorylation, FFA, protein synthesis, as well as purine and pyrimidine synthesis [82••]. The alterations in metabolic profiles in cancer cells help them to generate required anabolic components for cellular growth and proliferation during glycolytic stress. Coinhibition of glycolysis and mTORC1 activity in malignant cells attenuates their growth [82••]. These studies provide strong rationale for targeting metabolic pathways in malignant hematopoietic cells by inhibiting the PI3K-mTORC1 pathway.

\section{Conclusion}

From the various studies, discussed in this review, detailing the role of different components of PI3K-mTORC1 pathway, it can be inferred that they form a complex signaling mechanism, which regulates different processes essential for maintenance and functions of HSCs as well as LSCs. Given the importance of metabolic pathways in maintenance of HSCs and LSCs, it is important to further study the role of different components of PI3-K-mTORC1 pathway in regulating different metabolic processes in these cells. Moreover, using genetic approaches and specific pharmacological inhibitors, it is important to understand the role of each individual downstream substrates of mTORC1, S6K1, and 4E-BP1, in regulation of HSCs and LSCs. It is probable that downstream of mTORC1, these two molecules might regulate the HSC and LSC activities differentially. Moreover, as S6K1 negatively regulates Akt activation by inhibiting mTORC2 activity, it is critical to understand the effect of inhibition of S6K1 on HSCs and LSCs.

Although there have been extensive studies regarding the role of PI3K-mTORC1 pathway in HSCs, the role of this pathway in regulating the hematopoietic microenvironment has not been studied extensively. Current evidence suggests that PI3K-mTORC1 pathway plays a role in regulating the hematopoietic niche. In endothelial cells, activation of Akt results in increased frequency of functional HSCs along with an increase in engraftment potential [83]. Multiple studies have shown that most potent functional HSCs reside in the most hypoxic region of the bone marrow [84, 85]. In hypoxic conditions, HIF- $1 \alpha$ is a critical mediator of HSC quiescence and function [86, 87]. Given the complex interplay between HIF- $1 \alpha$ and PI3K-mTORC1 pathway [88-91], further studies are required to determine how this pathway regulates different components of the hypoxic hematopoietic niche and regulates hematopoiesis. Furthermore, leukemic cells interact with the hematopoietic microenvironment and alter the niche to support growth of malignant cells and impairing hematopoiesis. Mesenchymal stem cells (MSCs) derived from patients with myelodysplastic syndrome (MDS) display change in genetic signatures, which can be induced by MDS cells [92]. Development of myeloproliferative neoplasm (MPN) results in expansion of osteoblastic lineage cells (OBC) in the bone marrow [93]. Endothelial cells and osteoblasts also support the growth of leukemic cells [94, 95]. In human, malignant hematopoietic cells, interaction with bone marrow stromal cells promotes survival of LSCs [96]. Bone marrow stromal cells can also protect AML cells from targeted therapy by activating PI3K-mTORC1 pathway [97]. Also, multiple evidences suggest that dysregulation of signaling pathways in hematopoietic niche could also initiate leukemic transformation of HSCs [98-102]. To develop potential therapeutic interventions targeting the interaction between leukemic cells and bone marrow niche, it is important to determine the role of key signaling pathways that regulate the functions of leukemic hematopoietic cells as well as bone marrow microenvironment. Understanding the role of PI3K-mTORC1 pathway could provide new therapeutic targets to treat the deregulated hematopoietic microenvironment in human hematologic malignancies.

Acknowledgments This work was supported in part by grants from National Institutes of Health (R01HL077177 to RK; R01HL075816 to RK; R01HL081111 to RK, R01CA173852 to R. K; and R01CA134777 to $\mathrm{RK})$.

\section{Compliance with Ethical Standards}

Conflict of Interest Joydeep Ghosh and Reuben Kapur declare that they have no conflict of interest.

Human and Animal Rights and Informed Consent This article does not contain any studies with human or animal subjects performed by any of the authors.

\section{References}

Papers of particular interest, published recently, have been highlighted as:

-• Of major importance

1. Bowie MB et al. Hematopoietic stem cells proliferate until after birth and show a reversible phase-specific engraftment defect. J Clin Invest. 2006;116:2808-16.

2. Kiel MJ et al. Haematopoietic stem cells do not asymmetrically segregate chromosomes or retain BrdU. Nature. 2007;449:238-42.

3. Shlush LI et al. Identification of pre-leukaemic haematopoietic stem cells in acute leukaemia. Nature. 2014;506:328-33.

4. Lagadinou ED et al. BCL-2 inhibition targets oxidative phosphorylation and selectively eradicates quiescent human leukemia stem cells. Cell Stem Cell. 2013;12:329-41. 
5. Saito Y, Chapple RH, Lin A, Kitano A, Nakada D. AMPK protects leukemia-initiating cells in myeloid leukemias from metabolic stress in the bone marrow. Cell Stem Cell. 2015;17:585-96.

6. Kaizuka $\mathrm{T}$ et al. Ttil and Tel2 are critical factors in mammalian target of rapamycin complex assembly. J Biol Chem. 2010;285: 20109-16.

7. Zhao L, Vogt PK. Class I PI3K in oncogenic cellular transformation. Oncogene. 2008;27:5486-96.

8. Vanhaesebroeck B, Guillermet-Guibert J, Graupera M, Bilanges $\mathrm{B}$. The emerging mechanisms of isoform-specific PI3K signalling. Nat Rev Mol Cell Biol. 2010;11:329-41.

9. Kandel ES, Hay N. The regulation and activities of the multifunctional serine/threonine kinase Akt/PKB. Exp Cell Res. 1999;253: 210-29.

10. Vazquez $\mathrm{F}$ et al. Tumor suppressor PTEN acts through dynamic interaction with the plasma membrane. Proc Natl Acad Sci U S A. 2006;103:3633-8.

11. Vazquez F, Devreotes P. Regulation of PTEN function as a PIP3 gatekeeper through membrane interaction. Cell Cycle. 2006;5: 1523-7.

12. Das S, Dixon JE, Cho W. Membrane-binding and activation mechanism of PTEN. Proc Natl Acad Sci U S A. 2003;100: 7491-6.

13. Lai $\mathrm{KP}$ et al. S6K1 is a multifaceted regulator of Mdm2 that connects nutrient status and DNA damage response. EMBO J. 2010;29:2994-3006.

14. Inoki $\mathrm{K}, \mathrm{Li} \mathrm{Y}, \mathrm{Xu} \mathrm{T}$, Guan KL. Rheb GTPase is a direct target of TSC2 GAP activity and regulates mTOR signaling. Genes Dev. 2003;17:1829-34.

15. DeYoung MP, Horak P, Sofer A, Sgroi D, Ellisen LW. Hypoxia regulates TSC1/2-mTOR signaling and tumor suppression through REDD1-mediated 14-3-3 shuttling. Genes Dev. 2008;22:239-51.

16. Li Y, Inoki K, Yeung R, Guan KL. Regulation of TSC2 by 14-3-3 binding. J Biol Chem. 2002;277:44593-6.

17. Inoki $\mathrm{K}, \mathrm{Li} \mathrm{Y}, \mathrm{Zhu} \mathrm{T}$, Wu J, Guan KL. TSC2 is phosphorylated and inhibited by Akt and suppresses mTOR signalling. Nat Cell Biol. 2002;4:648-57.

18. Pullen $\mathrm{N}$ et al. Phosphorylation and activation of $\mathrm{p} 70 \mathrm{~s} 6 \mathrm{k}$ by PDK1. Science. 1998;279:707-10.

19. Isotani $\mathrm{S}$ et al. Immunopurified mammalian target of rapamycin phosphorylates and activates p70 S6 kinase alpha in vitro. J Biol Chem. 1999;274:34493-8.

20. Burnett PE, Barrow RK, Cohen NA, Snyder SH, Sabatini DM. RAFT1 phosphorylation of the translational regulators p70 S6 kinase and 4E-BP1. Proc Natl Acad Sci U S A. 1998;95:1432-7.

21. Liu P et al. Sin 1 phosphorylation impairs mTORC2 complex integrity and inhibits downstream Akt signalling to suppress tumorigenesis. Nat Cell Biol. 2013;15:1340-50.

22. Julien LA, Carriere A, Moreau J, Roux PP. mTORC1-activated S6K1 phosphorylates rictor on threonine 1135 and regulates mTORC2 signaling. Mol Cell Biol. 2010;30:908-21.

23. Greer EL et al. An AMPK-FOXO pathway mediates longevity induced by a novel method of dietary restriction in C. elegans. Curr Biol. 2007; 17:1646-56.

24. Brunet A et al. 14-3-3 transits to the nucleus and participates in dynamic nucleocytoplasmic transport. J Cell Biol. 2002;156:817-28.

25. Haneline LS et al. Genetic reduction of class IA PI-3 kinase activity alters fetal hematopoiesis and competitive repopulating ability of hematopoietic stem cells in vivo. Blood. 2006;107:1375-82.

26. Juntilla MM et al. AKT1 and AKT2 maintain hematopoietic stem cell function by regulating reactive oxygen species. Blood. 2010;115:4030-8.

27. Guo F et al. Mouse gene targeting reveals an essential role of mTOR in hematopoietic stem cell engraftment and hematopoiesis. Haematologica. 2013;98:1353-8.
28. Kalaitzidis D et al. mTOR complex 1 plays critical roles in hematopoiesis and Pten-loss-evoked leukemogenesis. Cell Stem Cell. 2012;11:429-39.

29. Magee JA et al. Temporal changes in PTEN and mTORC2 regulation of hematopoietic stem cell self-renewal and leukemia suppression. Cell Stem Cell. 2012;11:415-28.

$30 . \bullet$ Ghosh J et al. S6K1 regulates hematopoietic stem cell selfrenewal and leukemia maintenance. J Clin Invest. 2016;126:2621-5. This article provides a novel insight into the role of $\mathrm{S} 6 \mathrm{~K} 1$ in maintaining quiescence and function of HSCs and LSCs.

31. Chen C, Liu Y, Liu Y, Zheng P. mTOR regulation and therapeutic rejuvenation of aging hematopoietic stem cells. Sci Signal. 2009;2:ra75.

32. Luo Y et al. Rapamycin enhances long-term hematopoietic reconstitution of ex vivo expanded mouse hematopoietic stem cells by inhibiting senescence. Transplantation. 2014;97:20-9.

33. Rohrabaugh SL, Campbell TB, Hangoc G, Broxmeyer HE. Ex vivo rapamycin treatment of human cord blood CD34+ cells enhances their engraftment of NSG mice. Blood Cells Mol Dis. 2011;46:318-20.

34. Huang J, Nguyen- M, McCarty EO, Hexner G, Danet-Desnoyers PS, Klein. Maintenance of hematopoietic stem cells through regulation of Wnt and mTOR pathways. Nat Med. 2012;18:1778-85.

35. Yilmaz $\mathrm{OH}$ et al. Pten dependence distinguishes haematopoietic stem cells from leukaemia-initiating cells. Nature. 2006;441:475-82.

36. Lee JY et al. mTOR activation induces tumor suppressors that inhibit leukemogenesis and deplete hematopoietic stem cells after Pten deletion. Cell Stem Cell. 2010;7:593-605.

37. Tesio $\mathrm{M}$ et al. Pten loss in the bone marrow leads to G-CSFmediated HSC mobilization. J Exp Med. 2013;210:2337-49.

38. Kharas MG et al. Constitutively active AKT depletes hematopoietic stem cells and induces leukemia in mice. Blood. 2010;115:1406-15.

39. Chen $\mathrm{C}$ et al. TSC-mTOR maintains quiescence and function of hematopoietic stem cells by repressing mitochondrial biogenesis and reactive oxygen species. J Exp Med. 2008;205:2397-408.

40. Campbell TB, Basu S, Hangoc G, Tao W, Broxmeyer HE. Overexpression of Rheb2 enhances mouse hematopoietic progenitor cell growth while impairing stem cell repopulation. Blood. 2009;114:3392-401.

41. Ito $\mathrm{K}$ et al. PML targeting eradicates quiescent leukaemiainitiating cells. Nature. 2008;453:1072-8.

42. Rossi L et al. The tissue inhibitor of metalloproteinases 1 increases the clonogenic efficiency of human hematopoietic progenitor cells through CD63/PI3K/Akt signaling. Exp Hematol. 2015;43:974-85 e971.

43. Jia $\mathrm{Y}$ et al. Inositol 1,3,4,5-tetrakisphosphate negatively regulates phosphatidylinositol-3,4,5- trisphosphate signaling in neutrophils. Immunity. 2007;27:453-67.

44. Siegemund S et al. IP3 3-kinase B controls hematopoietic stem cell homeostasis and prevents lethal hematopoietic failure in mice. Blood. 2015;125:2786-97.

45. Chen C, Liu Y, Liu Y, Zheng P. Mammalian target of rapamycin activation underlies HSC defects in autoimmune disease and inflammation in mice. J Clin Invest. 2010;120:4091-101.

46. Essers MA et al. IFNalpha activates dormant haematopoietic stem cells in vivo. Nature. 2009;458:904-8.

47. Xu Q, Simpson SE, Scialla TJ, Bagg A, Carroll M. Survival of acute myeloid leukemia cells requires PI3 kinase activation. Blood. 2003;102:972-80.

48. Kubota Y, Ohnishi H, Kitanaka A, Ishida T, Tanaka T. Constitutive activation of PI3K is involved in the spontaneous proliferation of primary acute myeloid leukemia cells: direct evidence of PI3K activation. Leukemia. 2004;18:1438-40.

49. Chow S, Minden MD, Hedley DW. Constitutive phosphorylation of the S6 ribosomal protein via mTOR and ERK signaling in the 
peripheral blasts of acute leukemia patients. Exp Hematol. 2006;34:1183-91.

50. Chapuis $\mathrm{N}$ et al. Dual inhibition of PI3K and mTORC1/2 signaling by NVP-BEZ235 as a new therapeutic strategy for acute myeloid leukemia. Clin Cancer Res: An Off J Am Assoc Cancer Res. 2010;16:5424-35.

51. Chapuis $\mathrm{N}$ et al. Autocrine IGF-1/IGF-1R signaling is responsible for constitutive PI3K/Akt activation in acute myeloid leukemia: therapeutic value of neutralizing anti-IGF-1R antibody. Haematologica. 2010;95:415-23.

52. Kornblau SM et al. Simultaneous activation of multiple signal transduction pathways confers poor prognosis in acute myelogenous leukemia. Blood. 2006;108:2358-65.

53. Kornblau SM et al. Dynamic single-cell network profiles in acute myelogenous leukemia are associated with patient response to standard induction therapy. Clin Cancer Res: An Off J Am Assoc Cancer Res. 2010;16:3721-33.

54. Munugalavadla V, Sims EC, Borneo J, Chan RJ, Kapur R. Genetic and pharmacologic evidence implicating the p85 alpha, but not p85 beta, regulatory subunit of PI3K and Rac2 GTPase in regulating oncogenic KIT-induced transformation in acute myeloid leukemia and systemic mastocytosis. Blood. 2007;110:1612-20.

55. Munugalavadla V, Sims EC, Chan RJ, Lenz SD, Kapur R. Requirement for $\mathrm{p} 85$ alpha regulatory subunit of class IA PI3K in myeloproliferative disease driven by an activation loop mutant of KIT. Exp Hematol. 2008;36:301-8.

56. Sujobert $\mathrm{P}$ et al. Essential role for the p110delta isoform in phosphoinositide 3-kinase activation and cell proliferation in acute myeloid leukemia. Blood. 2005;106:1063-6.

57. Colamonici $\mathrm{M}$ et al. Dual targeting of acute myeloid leukemia progenitors by catalytic mTOR inhibition and blockade of the p110alpha subunit of PI3 kinase. Oncotarget. 2015;6:8062-70.

58. Cheong JW et al. Phosphatase and tensin homologue phosphorylation in the C-terminal regulatory domain is frequently observed in acute myeloid leukaemia and associated with poor clinical outcome. Br J Haematol. 2003;122:454-6.

59. Huang X, Li D, Li T, Zhao BO, Chen X. Prognostic value of the expression of phosphatase and tensin homolog and CD44 in elderly patients with refractory acute myeloid leukemia. Oncol Lett. 2015;10:103-10.

60. Tamburini J et al. Constitutive phosphoinositide 3-kinase/Akt activation represents a favorable prognostic factor in de novo acute myelogenous leukemia patients. Blood. 2007;110:1025-8.

61. Gao $\mathrm{Y}$ et al. Rheb1 promotes tumor progression through mTORC1 in MLL-AF9-initiated murine acute myeloid leukemia. J Hematol Oncol. 2016;9:36.

62. Hoshii T et al. mTORC1 is essential for leukemia propagation but not stem cell self-renewal. J Clin Invest. 2012;122:2114-29.

63. Nogami A et al. FLT3-ITD confers resistance to the PI3K/Akt pathway inhibitors by protecting the mTOR/4EBP1/Mcl-1 pathway through STAT5 activation in acute myeloid leukemia. Oncotarget. 2015;6:9189-205.

64. Naka $\mathrm{K}$ et al. TGF-beta-FOXO signalling maintains leukaemiainitiating cells in chronic myeloid leukaemia. Nature. 2010;463: 676-80.

65. Sykes $\mathrm{SM}$ et al. AKT/FOXO signaling enforces reversible differentiation blockade in myeloid leukemias. Cell. 2011;146:697-708.

66. Huang HL et al. DNA-damaging reagents induce apoptosis through reactive oxygen species-dependent Fas aggregation. Oncogene. 2003;22:8168-77.

67. Santos MA et al. DNA-damage-induced differentiation of leukaemic cells as an anti-cancer barrier. Nature. 2014;514:107-11.

68. Sujobert $\mathrm{P}$ et al. Co-activation of AMPK and mTORC1 induces cytotoxicity in acute myeloid leukemia. Cell Rep. 2015;11:1446-57.
69. Gustafson CL, Partch CL. Emerging models for the molecular basis of mammalian circadian timing. Biochemistry. 2015;54: 134-49.

70. Partch CL, Green CB, Takahashi JS. Molecular architecture of the mammalian circadian clock. Trends Cell Biol. 2014;24:90-9.

71. Puram RV et al. Core circadian clock genes regulate leukemia stem cells in AML. Cell. 2016;165:303-16.

72. Lipton JO et al. The circadian protein BMAL1 regulates translation in response to S6K1-mediated phosphorylation. Cell. 2015;161:1138-51.

73.• Takubo K et al. Regulation of glycolysis by Pdk functions as a metabolic checkpoint for cell cycle quiescence in hematopoietic stem cells. Cell Stem Cell. 2013;12:49-61. This article provides a thorough insite into metabolic requirements of HSC.

74. Behal RH, Buxton DB, Robertson JG, Olson MS. Regulation of the pyruvate dehydrogenase multienzyme complex. Annu Rev Nutr. 1993;13:497-520.

75. $\mathrm{Du} \mathrm{J}$ et al. Cited2 is required for the maintenance of glycolytic metabolism in adult hematopoietic stem cells. Stem Cells Dev. 2014;23:83-94.

76. Herst PM, Howman RA, Neeson PJ, Berridge MV, Ritchie DS. The level of glycolytic metabolism in acute myeloid leukemia blasts at diagnosis is prognostic for clinical outcome. $\mathrm{J}$ Leukoc Biol. 2011;89:51-5.

77. Tandon $\mathrm{P}$ et al. Requirement for ribosomal protein $\mathrm{S} 6$ kinase 1 to mediate glycolysis and apoptosis resistance induced by Pten deficiency. Proc Natl Acad Sci U S A. 2011;108:2361-5.

78. Barger JF et al. S6K1 determines the metabolic requirements for BCR-ABL survival. Oncogene. 2013;32:453-61.

79. M. Braun et al., CXCL12 promotes glycolytic reprogramming in acute myeloid leukemia cells via the CXCR4/mTOR axis. Leukemia, (2016).

80. Prasanna VK, Venkataramana NK, Dwarakanath BS, Santhosh V. Differential responses of tumors and normal brain to the combined treatment of 2-DG and radiation in glioablastoma. J Cancer Res Ther. 2009;5(Suppl 1):S44-7.

81. Raez LE et al. A phase I dose-escalation trial of 2-deoxy-Dglucose alone or combined with docetaxel in patients with advanced solid tumors. Cancer Chemother Pharmacol. 2013;71: 523-30.

82.• Pusapati RV et al. mTORC1-dependent metabolic reprogramming underlies escape from glycolysis addiction in cancer cells. Cancer Cell. 2016;29:548-62. The article describes the regulation of multiple metablic pathways by mTORC1 and how malignant cells escape from hlycolysis dependency.

83. Kobayashi $\mathrm{H}$ et al. Angiocrine factors from Akt-activated endothelial cells balance self-renewal and differentiation of haematopoietic stem cells. Nat Cell Biol. 2010;12:1046-56.

84. Parmar K, Mauch P, Vergilio JA, Sackstein R, Down JD. Distribution of hematopoietic stem cells in the bone marrow according to regional hypoxia. Proc Natl Acad Sci U S A. 2007;104: 5431-6.

85. Winkler IG et al. Positioning of bone marrow hematopoietic and stromal cells relative to blood flow in vivo: serially reconstituting hematopoietic stem cells reside in distinct nonperfused niches. Blood. 2010;116:375-85.

86. Forristal CE et al. Pharmacologic stabilization of HIF-1alpha increases hematopoietic stem cell quiescence in vivo and accelerates blood recovery after severe irradiation. Blood. 2013;121:759-69.

87. Takubo $\mathrm{K}$ et al. Regulation of the HIF-1alpha level is essential for hematopoietic stem cells. Cell Stem Cell. 2010;7:391-402.

88. Cheng SC et al. mTOR- and HIF-1alpha-mediated aerobic glycolysis as metabolic basis for trained immunity. Science. 2014;345:1250684.

89. Harada $\mathrm{H}$ et al. The Akt/mTOR pathway assures the synthesis of HIF-1alpha protein in a glucose- and reoxygenation-dependent manner in irradiated tumors. J Biol Chem. 2009;284:5332-42. 
90. Marhold M et al. HIF1alpha regulates mTOR signaling and viability of prostate cancer stem cells. Mol Cancer Res. 2015;13:556-64.

91. Agani F, Jiang BH. Oxygen-independent regulation of HIF-1: novel involvement of $\mathrm{PI} 3 \mathrm{~K} / \mathrm{AKT} / \mathrm{mTOR}$ pathway in cancer. Curr Cancer Drug Targets. 2013;13:245-51.

92. Medyouf $\mathrm{H}$ et al. Myelodysplastic cells in patients reprogram mesenchymal stromal cells to establish a transplantable stem cell niche disease unit. Cell Stem Cell. 2014;14:824-37.

93. Schepers K et al. Myeloproliferative neoplasia remodels the endosteal bone marrow niche into a self-reinforcing leukemic niche. Cell Stem Cell. 2013;13:285-99.

94. Bruserud O, Ryningen A, Wergeland L, Glenjen NI, Gjertsen BT. Osteoblasts increase proliferation and release of pro-angiogenic interleukin 8 by native human acute myelogenous leukemia blasts. Haematologica. 2004;89:391-402.

95. Hatfield K, Ryningen A, Corbascio M, Bruserud O. Microvascular endothelial cells increase proliferation and inhibit apoptosis of native human acute myelogenous leukemia blasts. Int J Cancer. 2006;119:2313-21.
96. Sison EA et al. MLL-rearranged acute lymphoblastic leukaemia stem cell interactions with bone marrow stroma promote survival and therapeutic resistance that can be overcome with CXCR4 antagonism. Br J Haematol. 2013;160:785-97.

97. Chen $\mathrm{P}$ et al. Bone marrow stromal cells protect acute myeloid leukemia cells from anti-CD44 therapy partly through regulating PI3K/Akt-p27(Kip1) axis. Mol Carcinog. 2015;54:1678-85.

98. Kim YW et al. Defective notch activation in microenvironment leads to myeloproliferative disease. Blood. 2008;112:4628-38.

99. Kode $\mathrm{A}$ et al. Leukaemogenesis induced by an activating betacatenin mutation in osteoblasts. Nature. 2014;506:240-4.

100. Raaijmakers $\mathrm{MH}$ et al. Bone progenitor dysfunction induces myelodysplasia and secondary leukaemia. Nature. 2010;464:852-7.

101. Walkley CR, Shea JM, Sims NA, Purton LE, Orkin SH. Rb regulates interactions between hematopoietic stem cells and their bone marrow microenvironment. Cell. 2007;129:1081-95.

102. Wang L et al. Notch-dependent repression of miR-155 in the bone marrow niche regulates hematopoiesis in an NF-kappaBdependent manner. Cell Stem Cell. 2014;15:51-65. 